\title{
Calidad de vida en el trabajo del personal de enfermería en una institución de segundo nivel de atención en Aguascalientes
}

\author{
Navarro-Rodríguez Diana Cristina *, Cheverría-Rivera Sofía ${ }^{*}$, , Múnera-Gaviria Hugo Alberto***
}

\section{Resumen}

- Objetivo: Describir la calidad de vida en el trabajo del personal

- de enfermería en una institución de segundo nivel de atención

- en Aguascalientes, México. Métodos: Estudio descriptivo, trans-

- versal en el que participaron 235 trabajadores de enfermería

- de un hospital público seleccionados por muestreo aleatorio

- simple durante el periodo de diciembre 2016-febrero 2017; se

- autoaplicó el instrumento CVT GOHISALO, constituido por 74

- items distribuidos en siete dimensiones, puntaje máximo 296

- puntos en escala tipo Likert de 0 a 4, confiabilidad por Alpha

- de Cronbach de 0.93; para el análisis de datos se utilizó el pro-

- grama estadístico SPSS V.2I; se contó con la aprobación de los

- Comités de Ética e Investigación y el consentimiento informado

- de cada participante. Resultados: El 73.6\% de los participantes

- fueron mujeres, edad promedio 39.3 años (DE=8.58), el 85. 1 \%

- fue personal asistencial, el 34.9\% perteneció al turno matutino,

- antigüedad laboral promedio I 3.7 años (DE=7.57). De manera

- global, el 49\% del personal de enfermería puntuó un nivel medio

- de calidad de vida en el trabajo, las dimensiones que pondera-

- ron alta calidad de vida en el trabajo fueron seguridad en el tra-

- bajo y desarrollo personal con el $46 \%$ y $39.1 \%$ respectivamente,

- en las cinco dimensiones restantes los encuestados presentaron

- baja calidad de vida en el trabajo. Conclusión: El personal de

- enfermería puntuó un nivel medio de calidad de vida en el tra-

- bajo, lo cual puede estar influenciado por la legislación laboral,

- el sistema sanitario y la división sexual del trabajo. LUXMÉDICA,

- AÑO 13, NÚMER0 39, SEPT-DIC 2018 PP 3-10.

- Palabras clave: calidad de vida, trabajo, personal de enfermería, salud laboral.

\section{Abstract}

Objective: To describe the quality of life at work of the nursing staff in a second-level care institution in Aguascalientes, Mexico. Methods: Descriptive, cross-sectional study in which 235 nursing workers from a public hospital were selected by simple random sampling from December 2016 to February 2017. The CVTGOHISALO instrument was self-applied, consisting of 74 items distributed in seven dimensions, with a maximum score of 296 points on a Likert scale of 0 to 4 , with a Cronbach's alpha reliability of 0.93; SPSS v.2I was used for the statistical data analysis. It was approved by the Research Ethics Committees and we had the informed consent for each participant. Results: $73.6 \%$ of the participants were women, with an average age of 39.3 years $(S D=8.58), 85.1 \%$ were staff nurses, $34.9 \%$ belonged to the morning shift, with average working years of 13.7 (SD =7.57). Overall, $49 \%$ of the nursing staff scored an average level of quality of life at work, the dimensions that weighted high quality of life at work were job safety and personal development with $46 \%$ and $39.1 \%$ respectively, in the remaining five dimensions, the respondents presented low quality of life at work. Conclusions: The nursing staff scored an average level of quality of life at work, which may be influenced by labor legislation, the health system and the sexual division of labor. LUXMÉDICA, AÑO 13, NÚMERO 39, SEPT-DIC 2018 PP 3-10.

Keywords: quality of life, work, nursing staff, occupational health

* Estudiante de la Maestría en Administración en Enfermería, Facultad de Enfermería, Universidad Autónoma de San Luis Potosí; México. E-mail: diananavarro06@hotmail.com

** Maestra en Administración de la Atención en Enfermería, profesora investigadora, Facultad de Enfermería, Universidad Autónoma de San Luis Potosí; México. E-mail: rivera_1403@hotmail.com

*** Magíster en Salud Colectiva, profesor investigador, Facultad de Enfermería, Universidad de Antioquía, Medellín; Colombia. E-mail: hugomunera@ yahoo.com

Fecha de recibido: 6 de febrero 2018

Fecha de aceptación: 15 de marzo 2018

Correspondencia MAE. Diana Cristina Navarro Rodríguez. Facultad de Enfermería y Nutrición de la Universidad Autónoma de San Luis Potosí. Avenida Niño Artillero \#130, Zona Universitaria, Código postal 78240 San Luis Potosí, México. Teléfono 444- 8262300, extensión 5069. Correo electrónico diananavarro_06@hotmail.com 


\section{Introducción}

La calidad de vida en el trabajo (CVT) se integra cuando el trabajador, a través del empleo ve cubiertas las necesidades personales de soporte institucional, seguridad en el trabajo, integración al puesto, satisfacción por el trabajo, bienestar conseguido a través de la actividad laboral, desarrollo personal y administración del tiempo libre. ${ }^{1}$ El interés por estudiarla se remonta a la Revolución Industrial, donde se iniciaron movimientos sociales para humanizar el entorno laboral, ${ }^{2}$ lo anterior también alcanzó a los servicios hospitalarios, ya que la nueva gestión pública los convirtió en empresas de mercado, que conllevaron a la sobrecarga de la tarea y la disminución del tiempo disponible, ${ }^{3}$ lo que puede comprometer el actuar profesional y la salud del personal de enfermería. ${ }^{4}$

En este sentido, una calidad de vida en el trabajo positiva se relaciona con el desempeño laboral, productividad, competitividad, realización personal y profesional, calidad de los cuidados y la salud física y psicológica del trabajador; ${ }^{5-9}$ en tanto que al deteriorarse se produce ausentismo, estrés, consumo de drogas y fármacos, alteraciones psicológicas, gastrointestinales, musculo-esqueléticas y Burnout. 5, 9-11

Desde esta perspectiva, es importante estudiar la CVT en enfermería, ya que es el primer contacto del sistema sanitario con la sociedad, razón por la que deben ser cuidados los factores físicos, psicológicos y ambientales del centro laboral, a fin de promover la satisfacción y salud del trabajador.

Actualmente la CVT en enfermería ha sido estudiada a través del instrumento CVT GOHISALO ${ }^{1}$ y en mayor proporción con el instrumento CVP 35 Validado por Cabezas en $1998 ;^{10}$ sin embargo este último no contempla las necesidades humanas básicas satisfechas a través del empleo. Diversos investigadores han encontrado variabilidad en la CVT del personal de enfermería debido al tipo de contratación al que pertenecen, siendo mejor en aquellos con contratación indefinida, adscritos a servicios hospitalarios ambulatorios, con mayor tiempo de trayectoria laboral, con menor número de horas dicadas a la jornada laboral y en el personal profesional. 5-6, 10,12-17

El artículo muestra la realidad que se vive en un hospital de segundo nivel de atención médica en el que aún no existe la tercerización de los servicios profesionales, donde se requiere de intervenciones curativas, conllevando a la generación de una relación totalmente o parcialmente compensatoria entre el paciente y el personal de enfermería.

El estudio tiene por objetivo describir la calidad de vida en el trabajo del personal de enfermería en una institución de segundo nivel de atención en Aguascalientes, México, debido al incremento del ausentismo no previsible registrado por el personal. 


\section{Material y métodos}

Estudio descriptivo transversal realizado en una institución pública de segundo nivel de atención médica en Aguascalientes, México, durante diciembre 2016-febrero 2017. La población de estudio fueron 546 trabajadores; se realizó un cálculo del tamaño de la muestra, considerando un nivel de confianza del $95 \%$ y un margen de error del $5 \%$, dando una muestra de 251 sujetos seleccionados a través de muestreo aleatorio simple.

Se incluyó al personal de enfermería asistencial y administrativo con contratación indefinida, de los diferentes turnos laborales y servicios hospitalarios; se excluyó al personal que se encontró de vacaciones, licencia o incapacidad durante el periodo de recolección.

Los datos sociodemográficos y laborales se recolectaron mediante una ficha de datos personales ex profeso. Para medir la calidad de vida en el trabajo se autoadministró el instrumento de Calidad de vida en el trabajo titulado CVT GOHISALO, de uso público, elaborado y validado en el año 2009 por González et al, ${ }^{1}$ en población mexicana del medio hospitalario; conformado por 74 ítems agrupados en 7 dimensiones (Soporte institucional, Seguridad en el trabajo, Integración al puesto, Satisfacción por el trabajo, Bienestar conseguido a través del trabajo, Desarrollo personal y Administración del tiempo libre), con puntaje máximo de 296 puntos en escala tipo Likert de 0 a 4, donde cero corresponde a nada satisfecho y cuatro a máxima satisfacción, con confiabilidad por Alpha de Cronbach de 0.93 .
Se proporcionó el consentimiento informado y los instrumentos en el área de trabajo al comienzo del turno y se recolectaron al final del mismo.

La recolección de los datos de la prueba piloto se llevó a cabo en otra institución de atención médica que reunió los mismos criterios de inclusión, durante las dos primeras semanas de noviembre del 2016, sin ser necesario realizar ajustes al instrumento.

El análisis de datos se realizó mediante el programa estadístico Statistical Package for the Social Science (SPSS) v.21, donde se empleó estadística descriptiva para las variables sociodemográficas, laborales y la CVT.

Para calificar de manera global la calidad de vida en el trabajo, se consideraron los siguientes puntos de corte: baja: 75183, media $=184-237$, alta $=238-292$, los cuales se obtuvieron a partir de los cuartiles de los valores resultantes de la muestra y para calificar las dimensiones, se tomó el baremo de los autores del instrumento, donde los puntajes obtenidos por debajo del valor T 40 indicaron baja CVT, del valor T 40 al T 60 media CVT y por arriba del valor T 60 alta CVT.

El estudio cumplió con las normas nacionales e internacionales de ética en investigación con seres humanos y se contó con la aprobación del Comité de Ética en Investigación de la Facultad de Enfermería de la Universidad Autónoma de San Luis Potosí y de la institución de salud participante, con los número de registro CEIFE-2016-188 y R-2016-101-16 respectivamente.

\section{| | | | | | | | | | | | | | | | | | | | | | | | | | | | | | | | | | | | | | | | | | | | | | | | | | | | | | | | | | | | | | | | | | | | | | | | | | | | | | | | | | | | | | | | | | | | | | | | | | || ||}

\section{Resultados}

Los resultados corresponden a 235 trabajadores, debido a que durante la recolección de los datos se obtuvieron 8 eliminaciones y 8 exclusiones. El $73.6 \%$ de los participantes fueron mujeres; $56.1 \%$ esta- ba casado. El grupo de edad predominante fue de 29 a 34 años representado por el $23.4 \%$, edad mínima 24 años y máxima 56 , en promedio 39.3 años $(\mathrm{DE}=8.58)$. El $45.5 \%$ contaba con carrera técnica. El $78.3 \%$ tenía hijos, en promedio 2 hijos; el 
$82.6 \%$ tenía dependientes económicos, en promedio 2 dependientes económicos.

En cuanto a la situación laboral, 85.1\% fue personal asistencial; $34.9 \%$ perteneció al turno matutino y $42.2 \%$ a los servicios de hospitalización. El 30.2\% tenían entre 2 y 7 años de antigüedad laboral, mínimo 2 años y máximo 28, en promedio 13.7 años ( $D E=7.57)$. El $85.5 \%$ contó con un solo empleo; $40.4 \%$ recibió como salario quincenal entre $\$ 5,000$ y $\$ 6,999$ MXN. La cantidad mínima de horas laboradas por semana fue 13 horas y máxima
120 , en promedio 61 horas $(D E=18.50)$.

Respecto a la CVT, el $46 \%$ tuvo alta calidad en la dimensión de Seguridad en el trabajo, al igual que el $39.1 \%$ en el Desarrollo personal; en las dimensiones restantes el personal mostró baja CVT, representado por el $51.8 \%$ en Soporte institucional, el $44.7 \%$ en Integración al puesto de trabajo, el $40.6 \%$ en Satisfacción por el trabajo, el $45 \%$ en Bienestar logrado a través del trabajo y el $57.4 \%$ en Administración del tiempo libre ( $\mathrm{Ta}$ bla 1).

\section{Tabla I}

Dimensiones de la calidad de vida en el trabajo percibida por el personal de enfermería

\begin{tabular}{|lccccccc}
\hline & \multicolumn{3}{c}{} & \multicolumn{2}{c}{$\mathrm{n}=235$} \\
\hline \multicolumn{1}{|c}{ Dimensión } & \multicolumn{2}{c}{ Alta } & \multicolumn{2}{c}{ Media } & \multicolumn{2}{c}{ Baja } \\
& $\mathrm{fr}$ & $\%$ & $\mathrm{fr}$ & $\%$ & $\mathrm{fr}$ & $\%$ \\
\hline Soporte institucional para el trabajo & 62 & 26.2 & 51 & 22.0 & 122 & 51.8 \\
\hline Seguridad en el trabajo & 108 & 46.0 & 80 & 34.0 & 47 & 20.0 \\
\hline Integración al puesto de trabajo & 45 & 19.3 & 85 & 36.0 & 105 & 44.7 \\
\hline Satisfacción por el trabajo & 71 & 30.4 & 69 & 29.0 & 95 & 40.6 \\
\hline Bienestar logrado a través del trabajo & 80 & 34.0 & 49 & 21.0 & 106 & 45.0 \\
\hline Desarrollo personal & 92 & 39.1 & 85 & 36.2 & 58 & 24.7 \\
\hline Administración del tiempo libre & 9 & 3.8 & 91 & 38.8 & 135 & 57.4 \\
\hline
\end{tabular}

El promedio global de la Calidad de vida que indica un nivel medio representado por en el trabajo fue de 199.6 ( $D E=41.48$ ), lo el 49\% del personal de enfermería (figura 1).

Percepción global de la calidad de vida en el trabajo del personal de enfermería

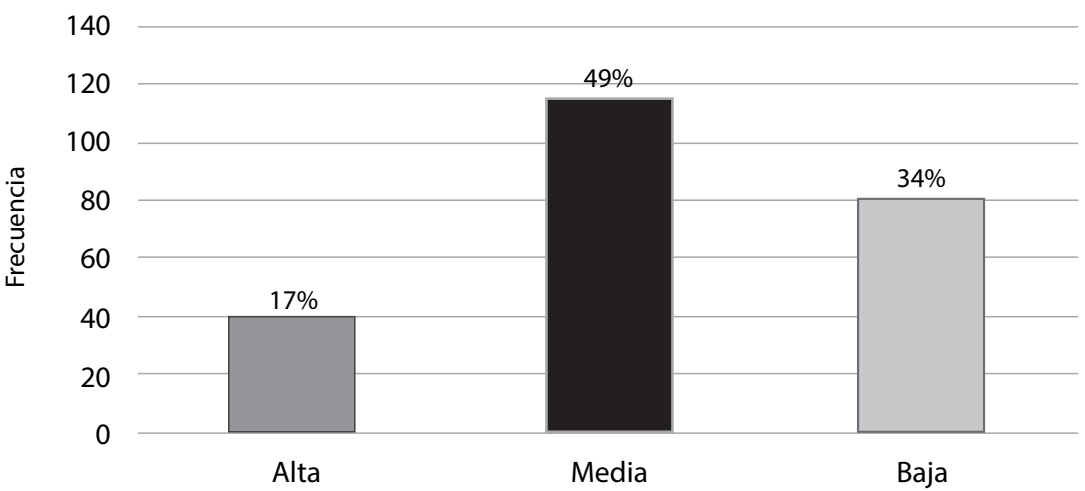

Nivel de calidad de vida en el trabajo 


\section{Discusión}

El sexo que predominó fue la mujer, lo que coincide con investigadores españoles ${ }^{10} y$ Latinoamericanos; ${ }^{5,12-16}$ en cuanto al estado civil, más de la mitad estaba casado y tenía hijos, similar a investigadores españoles, ${ }^{10}$ mexicanos ${ }^{13}$ y colombianos..$^{15}$

El promedio de edad fue de 39.3 años, lo que coincide con estudios colombianos ${ }^{15}$ y chilenos; ${ }^{16}$ el grado de estudios predominante fue la carrera técnica, en tanto que en el sector público ${ }^{5} \mathrm{y}$ privado de Mé$x_{\text {ico }}{ }^{12}$ y Chile ${ }^{16}$ prevaleció la licenciatura, para esta investigación las funciones que realiza el personal técnico difiere a las de otros países latinoamericanos, ya que dicho personal es responsable de la gestión de los servicios de enfermería.

Con relación a la situación laboral, predominó el personal asistencial del cuidado, del turno matutino (jornada laboral de 8 horas que sucede por la mañana) y de los servicios de hospitalización, lo que coincide con investigadores latinoamericanos. ${ }^{5,12-14}$

La antigüedad laboral promedio fue de 13.7 años, lo que difiere con investigadores mexicanos ${ }^{5,12}$ y chilenos, ${ }^{16}$ quienes reportaron una antigüedad menor a 5 años.

En cuanto al pluriempleo, más de la mitad contó con un solo trabajo, al igual que investigadores mexicanos, 12-13 colombianos ${ }^{15} \mathrm{y}$ chilenos; ${ }^{16}$ sin embargo, el hecho de contar con un solo empleo no significa que los recursos que obtienen a través de él son suficientes, sino que trabaja un mayor número de horas dentro de la misma institución para incrementar el ingreso económico, lo cual ha sido evidenciado en este estudio con 61 horas laboradas por semana, cantidad que es mayor a otras instituciones de México $^{13}$ y Colombia. ${ }^{15}$

Respecto al salario quincenal, cerca de la mitad recibió entre \$5, 000 y \$6, 999 MXN, cantidad que es menor a la de otras instituciones del sector salud mexicano, las cuales consideran a la profesionalización en el sueldo tabular, ${ }^{18,19}$ a su vez Colom- bia, ${ }^{20}$ España, Holanda y Alemania, ${ }^{21}$ tienen salarios más altos que en México. ${ }^{22}$

En cuanto a las dimensiones evaluadas, el Soporte institucional para el trabajo tuvo baja CVT, lo cual coincide con investigadores colombianos ${ }^{15} \mathrm{y}$ chilenos; ${ }^{16}$ por su parte investigadores peruanos ${ }^{14}$ encontraron puntaje medio, en tanto que otros investigadores mexicanos reportaron alta CVT. ${ }^{5}$, 12-13 Dicha dimensión evalúa los elementos del puesto de trabajo que soporta el empleo, tales como los procesos de trabajo, supervisión laboral, apoyo de los superiores, evaluación del trabajo, oportunidades de promoción y autonomía.

La dimensión de Seguridad en el trabajo tuvo alta CVT, similar a investigadores mexicanos, ${ }^{5,12}$ en tanto que otros investigadores del mismo país ${ }^{13}$ y de Perú14 reportaron puntajes medios; por su parte investigadores chilenos ${ }^{16}$ y colombianos $^{15}$ encontraron baja CVT. Dicha dimensión evalúa la firmeza de la relación del trabajador con la institución e incluye los procedimientos de trabajo, salarios, insumos, derechos contractuales y capacitación.

La dimensión de Integración al puesto de trabajo tuvo baja CVT, lo cual coincide con estudios de México, ${ }^{5,12-13}$ Perú, ${ }^{14}$ Colombia ${ }^{15}$ y Chile; ${ }^{16}$ dentro de los aspectos que evalúa incluye la pertinencia, motivación y ambiente de trabajo.

La dimensión de Satisfacción por el trabajo tuvo baja CVT, lo que coincide con investigadores mexicanos, ${ }^{5,12-13}$ peruanos, ${ }^{14}$ colombianos $^{15}$ y chilenos; $^{16}$ dicha dimensión evalúa el agrado respecto al empleo e incluye la dedicación al trabajo, orgullo por la institución, participación en el trabajo, autonomía, reconocimiento por el trabajo y autovaloración.

La dimensión de Bienestar logrado a través del trabajo, entendida como la satisfacción de las necesidades relacionadas con la manera de vivir, tuvo baja CVT, lo cual coincide con investigadores mexicanos, $^{5,}{ }^{12-13}$ peruanos $^{14}$ colombianos $^{15}$ y chilenos. ${ }^{16}$ Dicha dimensión incluye la sa- 
tisfacción por la vivienda, la salud general y la nutrición.

La dimensión de Desarrollo personal tuvo alta CVT, caso contrario a investigadores mexicanos, ${ }^{5,13}$ peruanos, ${ }^{14}$ colombianos ${ }^{15}$ y chilenos, ${ }^{16}$ quienes encontraron baja CVT; por su parte investigadores mexicanos Quintana $M$ y col ${ }^{12}$ reportaron un nivel medio de CVT; dicha dimensión alude al incremento de aspectos personales del trabajador relacionados con su actividad laboral y se valora mediante los logros, expectativas de mejora y seguridad personal.

La dimensión de Administración del tiempo libre tuvo baja CVT, lo que coincide con investigadores latinoamericanos: ${ }^{5,12,14-16} \mathrm{sin}$ embargo, los investigadores Quintana $M$ y coll $^{13}$ encontraron en el sector público de México alta CVT, dicha dimensión evalúa el disfrute de la vida en el horario en que no se realizan actividades laborales e incluye la administración del tiempo libre y el equilibrio entre el trabajo y la vida familiar.

\section{Conclusiones}

El personal de enfermería puntuó un nivel medio de Calidad de vida en el trabajo; sólo 2 de las 7 dimensiones tuvieron alta CVT: Seguridad en el trabajo y Desarrollo personal; las cinco dimensiones restantes tuvieron baja CVT.

Lo anterior representa un área de oportunidad para la gestión del talento humano y el fortalecimiento de la profesión, ya que la calidad de vida en el trabajo puede verse afectada por el incremento del tiempo de vida laboral de las nuevas reformas laborales en México, las cuales conllevarán a tener población envejecida en los centros de trabajo.

A su vez, deben ser consideradas las jornadas extensas de trabajo, ya que tienden a perjudicar la salud del personal, la seguridad del paciente y el tiempo destinado a la familia, factor que debe ser analizado por los gestores del cuidado, ya que un líder exitoso logra el equilibrio entre el cumplimiento de las metas y la preocupación de sus subordinados.

Es necesario seguir promoviendo el trabajo decente en la profesión, entendido como aquel que brinda salarios competitivos, seguridad social, equidad de género, formación profesional continua, fortalecimiento sindical y participación de los trabajadores, ${ }^{23}$ a través de la contratación indefinida del personal, ya que retiene la fuerza laboral. ${ }^{24}$ En este sentido, es de vital relevancia que exista literalidad entre la formación académica del personal y el puesto desempeñado, ${ }^{25}$ ya que previene la insatisfacción del trabajador y el desperdicio laboral.

Con base en lo anterior, se concluye que la calidad de vida en el trabajo del personal de enfermería puede estar influenciada por la legislación laboral y el sistema sanitario de salud, ${ }^{26}$ ya que tiene implicaciones sobre la turnicidad laboral y la carga de trabajo de la profesión, ${ }^{27}$ situación que debe ser considerada por los gestores de las instituciones sanitarias, ya que la doble carga de trabajo en las mujeres derivada de la división sexual, ${ }^{28}$ puede afectar la satisfacción del trabajador y propiciar el absentismo en enfermería. ${ }^{29}$

\section{Agradecimientos}

Al Consejo Nacional de Ciencia y Tecnología de México (CONACyT) por el apoyo otorgado con la beca mixta para la realización de este trabajo. 


\section{Bibliografía}

1. González Baltazar R, Hidalgo Santacruz G, Salazar Estrada JG, Preciado Serrano ML. Instrumento para medir la calidad de vida en el trabajo: CVT-GOHISALO: Manual para su aplicación e interpretación. México: Instituto de Investigación en Salud Ocupacional; 2009. ISBN: 978-970-764-866-1

2. Granados I. Calidad de vida laboral: Historia, dimensiones y beneficios. Rev investig psicol. [Internet] 2011;14(2):271-6. ISSN 1609 - 7445 [consultado 10 Agosto 2016] Disponible en: http://pepsic.bvsalud. org/pdf/ripsi/v14n2/a14.pdf.

3. Blanch JM. Calidad de vida laboral en hospitales y universidades mercantilizados. Pap psicol. [Internet] 2014;35(1):40-7. ISSN: 0214-7823[consultado 10 Junio 2017] Disponible en: http://www.redalyc.org/ articulo.oa?id=77830184006.

4. Quintana Zabala MO, Paravic Klijn TM. Calidad de vida en el trabajo del equipo de enfermería. Rev Bras Enferm. [Internet] 2014;67(2):302-5. doi:10.5935/0034-7167.20140041. [consultado 10 Marzo 2017] Disponible en: http://www.scielo.br/ pdf/reben/v67n2/0034-7167-reben-67-02-0302. pdf

5. Sánchez Guzmán MC, Carrillo ávila A. Calidad de vida laboral de los profesionales de enfermería del hospital general de Querétaro, SESEQ. [Tesis de licenciatura]. Querétaro: Universidad Autónoma de Querétaro; 2015. [consultado 17 Abril 2016]. Disponible en: http://ri.uaq.mx/bitstream/123456789/3526/1/ RI002497.pdf

6. Albanesi de Nasseta S. Percepción de la calidad de vida profesional en trabajadores de la salud. Altern en Psicol. [Internet]. 2013; (28): 8-19. [consultado 17 Abril 2016]. Disponible en: http://alternativas. $\mathrm{me} /$ index.php/numeros/28t/27-1-percepcion-decalidad-de-vida-profesional-en-trabajadores-de-lasalud

7. Moraes da Silva A, Magalhaes Guimaraes LA. Ocupational Stress and Quality of Life in Nursing. Paidéia. [Internet]. 2016; 26 (63)63-70. doi:10.1590/198243272663201608 [consultado 17 Abril 2016]. Disponible en: http://www.scielo.br/pdf/paideia/ v26n63/1982-4327-paideia-26-63-0063.pdf

8. Dimas Arias $M$, Ruiz Silva CM. ¿Cuál es la evidencia de que la calidad de vida laboral influye en las características del cuidado que brinda el personal de enfermería? Monográficos de investigación en salud. FORO I+E "Impacto social del conocimiento" - II Reunión Internacional de Investigación y Educación Superior en Enfermería - II Encuentro de Investigación de Estudiantes de Enfermería y Ciencias de la Salud. España; Noviembre 2015. Granada: Parainfo digital [Internet]. 2015. ISSN: 1988-3439 [consultado 17 Abril 2016]. Disponible en: http://www. index-f.com/para/n22/pdf/337.pdf.

9. Pérez Zapata D, Peralta Montecinos J, Fernández Dávila P. Influencia de las variables organizacionales en la calidad de vida laboral de funcionarios del sector público de salud en el externo norte de Chile. Univ Psychol [Internet]. 2013; 13 (2): 541-
51. doi:10.11144/Javeriana.UPSY13-2.ivoc [consultado 17 Abril 2016]. Disponible en: http://revistas.javeriana.edu.co/index.php/revPsycho/article/ view/4178

10. Blass Robledo M. Síndrome de Burnout y Calidad de Vida Profesional Percibida en matronas de Vizcaya (País Vasco) Evidentia. [Internet]. 2013; 10 (43). ISSN: 1697-638X [consultado 17 Abril 2016]. Disponible en: http://www.index-f.com/evidentia/n43/ ev8065r.php.

11. Sampaio Santos FA, Paiva Sousa $L$, Alves de Oliveira Serra MA, Chaves Rocha FA. Factors that influence the quality of life of community health workers. Acta Paul Enferm. [Internet]. 2016; 29(2):191-7. doi: http://dx.doi.org/10.1590/1982- 0194201600027 [consultado 15 Julio 2017]. Disponible en: http:// www.scielo.br/pdf/ape/v29n2/en_1982-0194ape-29-02-0191.pdf

12. Quintana Zavala MO, Sáez Carrillo K, Figueroa Ibarra C, García PugaJA, Salazar Ruibal RE, Tijanero González RM. Calidad de vida laboral de enfermeras de un hospital privado. Biotecnia. [Internet]. 2016;18(2):34-8. [consultado 29 Mayo 2017]. Disponible en: biotecnia.unison.mx/index.php/biotecnia/article/download/226/183.

13. Quintana Zaval MO, Paravic Klijm T. Calidad de vida en el trabajo, personal de enfermería. Secretaría de salud pública, Hermosillo-México. [Tesis de doctorado]. Chile: Universidad de Concepción; 2014. [consultado 17 Abril 2016]. Disponible en: http:// repositorio.udec.cl/bitstream/handle/11594/1603/ Tesis_Calidad_de_Vida_en_el_Trabajo.Image.Marked.pdf?sequence $=1$.

14. Peñarrieta de Córdova I, Santiago Abregú S, Krederdt Raujo S, Guevara Morote G, Carhuapoma Acosta M, Chávez Flores E. Validación del instrumento: Calidad de vida en el trabajo CVT-GOHISALO en enfermería del primer nivel de atención. Rev Enferm Herediana. [Internet]. 2014;7(2):124-31. ISSN: 19985487. [consultado 29 Mayo 2017]. Disponible en: http://www.upch.edu.pe/faenf/images/pdf/Revistas/2014/julio/Especial 3-7-2.pdf

15. Garcés Rodríguez AO, Lozada MA. Calidad de vida en el trabajo del profesional médico y de enfermería en una empresa social del estado (E.S.E) III Nivel, Bogotá. [Tesis de maestría]. Colombia: Universidad Nacional de Colombia; 2014. [consultado 27 Junio 2017]. Disponible en: http://www.bdigital.unal.edu. co/40120/1/539516.2014.pdf.

16. Delgado García D, Inzulza González M, Delgado García F. Calidad de vida en el trabajo: Profesionales de la salud de Clínica Río Blanco y Centro de Especialidades Médicas. Med Segur Trab. [Internet] 2012;58(228):216-23. ISSN: 1989 - 7790 [consultado 29 Mayo 2017]. Disponible en:http://scielo.isciii. es/pdf/mesetra/v58n228/original3.pdf.

17. Hermosilla Ávila A, Paravic Klijn T, Valenzuela Suazo S. Fuerza Laboral que Envejece, ¿Qué Hacer ante esta Tendencia? Cienc y Trab. [Internet] 2015;(54):16670. [consultado 29 Mayo 2017]. Disponible en: http://www.scielo.cl/pdf/cyt/v17n54/art02.pdf.

18. de Cássia de Marchi R, Almeida da Silva L, Olivei- 
ra Cruz AM, do Carmo Cruz ML. Carga horaria de trabajo de los enfermeros y su relación con las reacciones fisiológicas de estrés. Rev Latino-Am Enferm. [Internet] 2014;22(6):959-65. doi:10.1590/01041169.3292.2503 [consultado 29 Mayo 2017]. Disponible en: http://www.scielo.br/pdf/rlae/v22n6/ es_0104-1169-rlae-22-06-00959.pdf

19. Secretaría de Salud. Subsecretaría de Administración y Finanzas. Tabulador Rama Médica, Paramédica y Grupo afín. México: Secretaría de Salud; 2016. [consultado 20 Junio 2017] Disponible en: http://webcache.googleusercontent. com/search?q=cache:http://i.guerrero.gob.mx/ uploads/2016/10/DGRH-5194-2016-TABULADOR-UNICO-2016.pdf

20. Mateus Tobasura P, Polanía Restrepo SP, Rodríguez Vargas MA, Nelson Román R. Asignación salarial en los profesionales de enfermería y medicina general de Colombia. [Tesis de especialidad]. Colombia: Universidad Católica de Manizales; 2016. [consultado 20 Junio 2017] Disponible en: http://200.21.94.179:8080/jspui/bitstream/handle/10839/1516/Paola\% 20Mateus\% 20Tobasura. pdf? sequence $=1$ \&isAllowed $=y$

21. Fuentes ML. México social: enfermería, la relevancia de hacer más. Excelsior. [Internet]. 2015. [consultado 20 Junio 2017] Disponible en: http://www.excelsior.com.mx/nacional/2015/01/13/1002361

22. Serrano Orellana BJ, Portalanza A. Influencia del liderazgo sobre el clima organizacional. Suma Neg. [Internet] 2014;5(11):117-25. [consultado 29 Mayo 2017]. Disponible en: http:// ac.els-cdn.com/S2215910X14700266/1-s2.0S2215910X14700266-main.pdf? tid =c6deac163989-11e7-b62b-00000aab0f26\&acdnat $=149486$ 5136 741960cfc6e9b6a03ebddab17c94f0d9.

23. Quintana Zavala MO, Valenzuela Suazo S, Paravic Klijn T. Enfermería desde la perspectiva del Trabajo Decente. Enferm. glob. [Internet] 2014;(33):302-9. ISSN: 1695-6141 [consultado 29 Mayo 2017]. Disponible en: http://scielo.isciii.es/pdf/eg/v13n33/ ensayo1.pdf.

24. Trapp A, Larrain AI, Santis MJ, Olbrich S. Causas de abandono de la práctica clínica hospitalaria de enfer- mería Cienc. enferm. [Internet] 2016;22(2):39-50. doi:10.4067/S0717-95532016000200004. [consultado 29 Mayo 2017]. Disponible en: http://www. scielo.cl/scielo.php?script $=$ sci_arttext\&pid $=$ S0717 95532016000200004\&lng $=e^{-} \& n r m=i s o \& t \operatorname{lng}=$ en

25. Barbera Ortega MC, Cecagno D, Seva Llor AM, Heckler de Siqueira HC, López Montesinos MJ, Maciá Soler L. Formación académica del profesional de enfermería y su adecuación al puesto de trabajo. Rev Latino-Am Enferm. [Internet] 2015;23(3):404-10. doi:10.1590/0104-1169.0432.2569. [consultado 29 Mayo 2017]. Disponible en: http://www.scielo.br/ pdf/rlae/v23n3/es_0104-1169-rlae-23-03-00404. pdf

26. Pereyra F, Micha A. La configuración de las condiciones laborales de la enfermería en el Área Metropolitana de Buenos Aires: Un análisis en el cruce del orden de género y la organización del sistema de salud. Salud Colectiva. [Internet] 2016;12(2):221 38. ISSN: 1669-2381. doi: 10.18294/sc.2016.730. [consultado 29 Mayo 2017]. Disponible en: http:// www.redalyc.org/articulo.oa?id=73146051006

27. Gago López $M M$, Bouzada Rodríguez $A L$, Otero López A, Otero López C, Calvo Alonso J, Carracero Martín R. El trabajo a turnos. Una realidad en la vida y la salud de las enfermeras. Nure Investig. [Internet] 2013;10(64):1-23.ISSN: 1697-218X [consultado 29 Mayo 2017]. Disponible en: http://www. nureinvestigacion.es/OJS/index.php/nure/article/ view/624/613.

28. Mosqueda Díaz A, Paravic Klijn T, Valenzuela Suazo S. División sexual del trabajo y Enfermería. Indez Enferm. 2013;22(1):70-4. doi: dx.doi, org/10.4321/S1132-12962013000100015 [consultado 29 Mayo 2017]. Disponible en: http:// scielo.isciii.es/scielo.php?script $=$ sci_arttext\&pid $=$ S1132-12962013000100015

29. Blanca Gutiérrez JJ, Jiménez Díaz MC, Escalera Franco LF. Intervenciones eficaces para reducir el absentismo del personal de enfermería hospitalario. Gac Sanit. [Internet]. 2013;27(6):545-51.doi: http:// dx.doi.org/10.1016/j.gaceta.2012.09.006 [consultado 29 Mayo 2017]. Disponible en: http://scielo. isciii.es/pdf/gs/v27n6/revision.pdf 